Michel Bouvier

\title{
LA NOUVELLE GOUVERNANCE FINANCIÈRE DE L'ETAT EN FRANCE
}

Le Parlement a réformé à son initiative ${ }^{1}$, par une loi du $1^{\text {er }}$ août 2001 , l'ordonnance du 2 janvier 1959 portant loi organique relative aux lois de finances ${ }^{2}$ qui organisait jusqu'alors le cadre budgétaire de l'Etat. Cette réforme est doublement importante. Elle l'est par son objet puisque c'est en effet la «constitution financière » de la France qui se trouve concernée. Elle l'est par son ampleur puisque le droit budgétaire de l'Etat se trouve remanié en profondeur³.

Au regard de l'histoire du droit public financier comme de celle des institutions politiques, le texte marque à l'évidence une étape importante. Ce n'est pas seulement d'une adaptation technique dont il s'agit. Plus largement, c'est un nouveau contrat social pour les finances publiques, socle d'une réforme de l'Etat qui est en filigrane du nouveau dispositif. Celui-ci ne se contente pas, en effet, de redéfinir les rapports entre Parlement et gouvernement en augmentant de manière notable les pouvoirs d'initiative et de contrôle des députés et des sénateurs, alors que l'ordonnance de 1959, dans la logique de la Constitution de 1958, avait eu au contraire pour objectif de renforcer ceux du gouvernement.

Tournant le dos à la classique logique de moyens qui s'attache à ne considérer que le montant des crédits alloués, la LOLF, qui s'applique pleinement depuis le $1^{\text {er }}$ janvier 2006, lui substitue une logique de résultats qui, marquée par une philosophie d'entreprise, prend d'abord en considération les objectifs à atteindre. Ce nouveau paradigme entraîne des bouleversements en profondeur de la gestion publique et partant de l'organisation interne des administrations.

1 Proposition «Migaud» du 11 juillet 2000. II convient de souligner que, contrairement au passé, l'initiative n'est pas venue de l'exécutif. Cf les principaux enjeux de la réforme in Revue Française de Finances Publiques $\mathrm{N}^{\circ} 73-$ 2001 :Réforme des finances publiques : Réforme de l'Etat.

$2 \quad$ Loi $N^{\circ}$ 2001-692 publiée au JO du 2 août 2001 et validée par le Conseil Constitutionnel (décision $N^{\circ} 2001-$ 448 DC du 25 juillet 2001). Pour une présentation exhaustive de la loi , cf. RFFP N 76-2001: La loi organique relative aux lois de finances ; cf également RFFP $N^{\circ} 82-2003$ : Mettre en œuvre la LOLF et RFFP $N^{\circ} 87-2004$ : Finances publiques : enjeux pour l'avenir ;aussi RFFP N`93-2005 : Le budget 2006 en LOLF. Pour approfondir, cf. M. Bouvier, MC Esclassan, JP Lassale, Manuel de Finances publiques, Editions LGDJ 2008, 9ème édition. Qui a été adoptée par le Sénat en deuxième lecture le 28 juin 2001 (292 voix pour, 17 voix contre). 


\section{Les origines de la LOLF}

\section{La LOLF s'inscrit dans un processus historique de réforme des Finances publiques}

La LOLF n'est en rien un texte ponctuel. Elle s'inscrit dans un processus de réforme des Finances publiques et de l'Etat qui a débuté, dans la seconde moitié des années 1970, par une critique théorique de l'impôt et de la dépense publique. Ce processus s'est poursuivi de manière très concrète à partir de la seconde moitié des années 1980 non seulement par des mesure allant dans le sens d'un allègement de la pression fiscale pesant sur les entreprises, mais aussi par des mesures structurelles telles que les privatisations ou la décentralisation.

Ce sont bien, à notre sens, les évolutions des trente dernières années qui donnent tout son sens à la réforme budgétaire actuelle. Cette période est en effet celle au cours de laquelle s'est amorcé un processus portant en germe une véritable métamorphose des systèmes financiers publics, et partant de l'Etat, une période dominée par ailleurs sur le fond par la généralisation d'une conception beaucoup plus libérale de la société et traversée enfin par des réformes institutionnelles d'ampleur comme la décentralisation, la monnaie unique européenne ou encore le développement spectaculaire des finances sociales.

On ne s'étonnera pas que dans ce contexte les limites de l'ordonnance du 2 janvier 1959, l'ancien droit budgétaire de l'Etat, aient pu se faire de plus en plus évidentes au fur et à mesure que celle-ci s'est trouvée confrontée à des réalités nationales et internationales fort différentes de celles qui, à l'origine, avaient présidé à l'élaboration du texte. Prise dans le cadre de la fin des années 1950 dominées par une conception très centralisatrice et très interventionniste de l'État, l'ordonnance en portait incontestablement les marques, reflétant les préoccupations et les illusions de la société d'après-guerre, alors qu'elle avait continué à s'appliquer à un environnement dans lequel des valeurs souvent inverses avaient pris le pas.

\section{La LOLF s'inscrit dans un processus politique et sociologique}

Les raisons de la réforme ne se limitent pas toutefois à la seule obsolescence d'un texte ni même, à vrai dire, à la seule situation française qui n'a rien de bien singulier. Ses causes sont en réalité complexes; elles ne sont pas seulement liées à des nécessités économiques et financières, mais également au fait que le contexte politique français a changé avec notamment la volonté du pouvoir législatif d'accroître son pouvoir de décision financiére ainsi que la prise de conscience de l'ensemble de la classe politique de la nécessité de réformer les procédures budgétaires. 
A la différence de l'ordonnance de 1959 et des textes financiers qui l'ont précédée, le caractère consensuel du processus d'élaboration de ce document est tout à fait original; il s'agit d'un processus d'initiative législative dont l'issue était pourtant loin d'être évidente si l'on se reporte aux termes dans lesquels le rapporteur général de la commission des finances de l'Assemblée nationale, Didier Migaud, s'adressait aux députés le 8 février 2000: " Nous devons aboutir! Mais nous ne le pourrons que si nous sommes d'accord à trois: Assemblée nationale, Sénat et Gouvernement. C'est le vœu que je forme ». De ce point de vue c'est donc avec un sens de l'intérêt général et des responsabilités particulièrement poussé que la classe politique a pu, avec l'aide des fonctionnaires concernés, mener à son terme la réforme et dessiner ainsi une nouvelle architecture financière pour l'Etat . On pourra même retenir plus tard que l'adoption de ce texte fut, selon l'expression d'Alain Lambert, alors président de la commission des finances du Sénat, «un moment d'exception et d'excellence... un acte majeur de maturité démocratique... », un acte par lequel des personnalités très différentes ont eu «le génie de s'accorder pour redonner sens à la démocratie et redonner vie et force à leur Etat ».

En même temps, c'est sans doute aussi le contexte sociologique qui s'est modifié, on veut dire le regard porté par les citoyens sur les dépenses et les recettes publiques. L'argent se faisant rare ils se montrent aujourd'hui indéniablement plus réceptifs à la question du contrôle des deniers publics, plus intéressés qu'autrefois par l'usage qui en est fait, plus sensibles donc au thème du contrôle de la dépense et à son corollaire, l'utilisation des prélèvements obligatoires. Il est à observer de même que dans les attentes à ce sujet, deux logiques viennent dorénavant se côtoyer : la première, d'essence plutôt politique place au premier plan la transparence financière, la lisibilité des budgets et des comptes publics; la seconde, d'essence plutôt économique, et qui se montre quant à elle essentiellement préoccupée par la rationalisation, l'efficacité et la performance de la gestion de l'argent public favorise la montée d'une culture du contrôle et de la gestion des fonds publics et cela au-delà même du cercle retreint des décideurs politiques et des gestionnaires.

\section{La LOLF s'inscrit dans un processus international de restructuration des systèmes financiers publics}

La mondialisation des échanges, qui s'est considérablement intensifiée ces dernières années a pour conséquence que, du point de vue des finances publiques, les systèmes financiers publics des Etats peuvent, à juste titre, être considérés comme des système à risques et la situation avtuelle en est une démonstration parfaite. .

La nécessité d'un pilotage adéquat de tels systèmes exige que non seulement un langage commun à l'ensemble des acteurs soit utilisé mais aussi qu'ils respectent une logique commune de fonctionnement, des normes communes. Autrement dit, il est fondamental qu'ils partagent une même logique juridique et de gestion et il est 
nécessaire que celle-ci soit parfaitement intériorisée, intégrée dans leur mode de fonctionnement afin qu'une autodiscipline du système devienne possible.

C'est bien la voie dans laquelle se sont engagés la plupart des Etats ces dernières années avec l'invitation qui leur a été faite par le FMI ou l'OCDE ${ }^{4}$ d'adhérer à des codes de bonne conduite ou mieux, comme dans le cadre de l'UE ,d'intégrer dans leur législation interne un certain nombre de normes contraignantes et de les respecter. En d'autres termes, la réorganisation du système financier international et la prévention des risques passe aussi, et peut-être surtout, par la réorganisation des systèmes nationaux de gestion des finances publiques, caractériséé notamment par la mise en place de standards internationaux de gestion financière.

\section{Une conception stratégique de l'action de l'Etat et de la prise de décision financière publique}

\section{Une nouvelle présentation des crédits par objectifs : missions, programmes, dotations}

La LOLF redéfinit d'abord de manière radicale la présentation du budget et par là même, l'étendue des pouvoirs financiers du Parlement. Ceux-ci portant prioritairement sur la répartition et l'utilisation des crédits en fonction d'objectifs préalablement fixés, le Parlement ne se borne donc plus comme auparavant à faire des choix en termes de moyens . La nouvelle architecture budgétaire s'établit par missions (environ 50) qui elles-mêmes regroupent des programmes (environ 150), nouvelles unités de répartition des crédits; la nouvelle structure n'a pas seulement pour effet de donner plus de « lisibilité » au document budgétaire et par conséquent peut être plus d'attrait du fait d'une meilleure visibilité de l'action publique et de ses enjeux financiers; elle favorise une meilleure cohérence de l'action publique en évitant un trop grand fractionnement des politiques publiques dans l'espace et dans le temps.

\section{Les missions ministérielles ou interministérielles : des unités de vote des crédits}

Selon les termes de la loi, les crédits ouverts par les lois de finances pour couvrir chacune des charges budgétaires de l'Etat sont regroupés par mission relevant d'un ou plusieurs services d'un ou plusieurs ministères. Chaque budget annexe de même que chacun des comptes spéciaux représente également une mission (leurs crédits étant spécialisés par programme) .

4 Cf sur ce point, Michel Bouvier, La surveillance multilatérale internationale des finances publiques et le pouvoir politique, in Processus budgétaire,vers un nouveau rôle du Parlement, Ed Sénat/OCDE 2002. 
Il s'ensuit que des programmes ayant la même finalité sont regroupés en missions qui, elles, peuvent être interministérielles. La mission, qui ne peut être créée que par une disposition d'une loi de finances d'initiative gouvernementale, comprend ainsi un ensemble de programmes concourant à une politique publique définie.

La mission représente par ailleurs l'unité de vote. Le vote des crédits s'effectuant par mission, il intervient par conséquent sur des objectifs ministériels ou interministériels.

Répartition des crédits du budget général et des emplois par mission (2009-2011)

\begin{tabular}{|c|c|c|c|c|c|c|}
\hline \multirow[b]{2}{*}{ En miliards d'ouros } & \multicolumn{2}{|c|}{2009} & \multicolumn{2}{|c|}{2010} & \multicolumn{2}{|c|}{2011} \\
\hline & $\begin{array}{l}\text { Autorisations } \\
\text { dengagement }\end{array}$ & Crédits de paiement & $\begin{array}{l}\text { Autorisations } \\
\text { dengagement }\end{array}$ & Crédits de paiement & $\begin{array}{l}\text { Autorisations } \\
\text { d'engagement }\end{array}$ & Crédits de paiement \\
\hline Action exterieure de l'Etat & 2,50 & 2,52 & 2,58 & 2,55 & 2,50 & 2,52 \\
\hline Administration generale et territoriale de rétat & 2,61 & 2,60 & 2,63 & 2,63 & 2,56 & 2,56 \\
\hline Agriculture, peche, foret et affaires rurales & 3,24 & 3,49 & 2,93 & 3,16 & 2,92 & 3,03 \\
\hline Aide publique au developpement & 3,38 & 3,17 & 2,85 & 3,24 & 4,43 & 3,24 \\
\hline Anciens combattants, memoire et liens avec la Nation & 3,55 & 3,53 & 3,44 & 3,45 & 3,34 & 3,34 \\
\hline Conseil et controle de l'Etat & 0,55 & 0,55 & 0,57 & 0,57 & 0,59 & 0,59 \\
\hline Culture & 2,84 & 2,78 & 2,72 & 2,80 & 2,72 & 2,82 \\
\hline Defense & 4779 & 37,39 & 37,00 & 38,06 & 37,76 & 38,72 \\
\hline Direction de l'action du Gouvernement & 0,49 & 0,54 & 0,52 & 0,51 & 0,53 & 0,51 \\
\hline Eoologie, developpernent et amenagement durables & 10,25 & 10,07 & 10,25 & 10,20 & 9,39 & 9,34 \\
\hline Eoonomie & 1,91 & 1,90 & 1,93 & 1,92 & 1,94 & 1,93 \\
\hline Engagements financiers de l'Etat & 46,00 & 46,00 & 4744 & 47,44 & 49,40 & 49,40 \\
\hline Enseignement scolaire & 60,01 & 59,99 & 61,67 & 61,65 & 62,95 & 62,93 \\
\hline Gestion des finences publiques et des ressources hurnaines & 11,63 & 11,37 & 11,39 & 11,54 & 11,41 & 11,53 \\
\hline Imrnigration, asie et integration & 0,51 & 0,51 & 0,51 & 0,51 & 0,51 & 0,51 \\
\hline Justice & 8,32 & 6,65 & 7,14 & 6,94 & 2,10 & 7,04 \\
\hline Medias & 1,02 & 1,01 & 1,01 & 1,00 & 0,99 & 0,99 \\
\hline Outre-mer & 1,97 & 1,88 & 2,00 & 1,93 & 2,00 & 1,93 \\
\hline Politique des territoires & 0,39 & 0,37 & 0,35 & 0,38 & 0,32 & 0,38 \\
\hline Pouwoirs publics & 1,05 & 1,05 & 1,06 & 1,06 & 1,07 & 1,07 \\
\hline Provisions & 0,23 & 0,23 & 0,68 & 0,66 & 1,15 & 1,15 \\
\hline Recherche et enseignernent superieur & 24,56 & 24,16 & 25,45 & 24,96 & 26,27 & 25,87 \\
\hline Regines sociaux et de retraite & 5,18 & 5,18 & 5,45 & 5,45 & 5,75 & 5,75 \\
\hline Relations avec les collectivites territoriales & 2,41 & 2,34 & 2,46 & 2,40 & 2,51 & 2,44 \\
\hline Sante & 1,13 & 1,16 & 1,15 & 1,17 & 1,17 & 1,19 \\
\hline Securite & 16,16 & 16,23 & 16,71 & 16,63 & 1727 & 1700 \\
\hline Securite civile & 0,45 & 0,42 & 0,41 & 0,42 & 0,45 & 0,43 \\
\hline Solidarite, insertion et egalite des chances & 11,20 & 11,18 & 11,58 & 11,60 & 12,13 & 12,15 \\
\hline Sport, jeunesse et vie associative & 0,80 & 0,79 & 0,75 & 0,77 & 0,73 & 0,75 \\
\hline Tiavail et muplui & $11, \pi 0$ & 11,02 & 10,74 & 10,74 & 10,00 & 10,51 \\
\hline Ville et logement & 260 & 7,64 & 730 & 7,53 & 728 & 7,37 \\
\hline Total & 291,48 & 278,50 & 282,66 & 283,89 & 289,77 & 289,00 \\
\hline
\end{tabular}

\section{Les programmes}

La notion de programme exprime le passage d'une culture de moyens à une culture de résultat

Le programme représente la clef de la réforme dès lors que posant en principe une budgétisation des crédits par objectifs, il conduit les décideurs publics, politiques ou gestionnaires, à cesser de raisonner strictement en termes de moyens. Selon la LOLF, les crédits destinés à réaliser une action ou un ensemble cohérent d'actions (environ 600) relevant d'un même ministère sont regroupés sous la forme d'un programme, étant entendu que ces actions sont associées ou participent à des objectifs définis en fonction de finalités d'intérêt général . et que les résultats doivent faire ensuite l'objet d'une évaluation pratiquée au moyen d'indicateurs de performance. 
La portée de la mesure est claire. Au classique budget de moyens succède donc un budget de résultats, c'est à dire un budget dans lequel les crédits ne sont plus présentés par nature de dépenses, mais par objectifs et donc selon une démarche qui met au premier plan la notion de performance de la dépense publique. Telle est la voie par laquelle le législateur a entendu introduire une plus grande rationalité dans la gestion financière publique. Les décideurs sont obligés de définir d'abord des politiques d'action en vue de l'obtention des crédits nécessaires. Ils doivent par ailleurs, une fois l'opération effectuée, en évaluer l'efficacité. S'y ajoute que le dispositif permet également de mesurer l'utilité de la dépense dès lors que cette dernière n'est plus fonction de la nature des crédits alloués mais d'objectifs clairement définis et de résultats attendus.

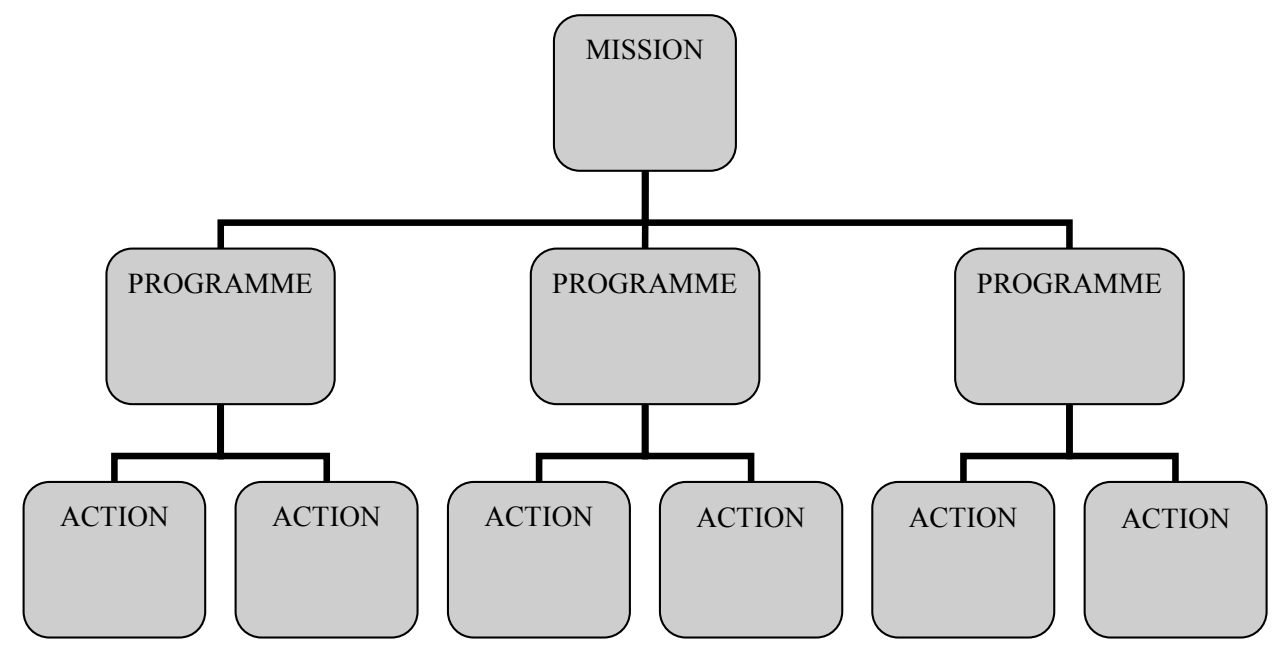

\section{Les programmes sont des unités de spécialité}

Les crédits sont spécialisés par programme.Avec cette nouvelle unité de spécialité, s'exprime indiscutablement un souci de cohérence et de simplification de la nouvelle nomenclature budgétaire . Celle-ci, qui est complètement transformée, comporte de 150 à 170 programmes, soit une réduction appréciable si l'on songe que les crédits étaient jusqu'à 2005 dispersés en 850 chapitres. Les programmes se déclinent ensuite en actions et sous-actions.

\section{Les programmes sont circonscrits par ministère}

Certains auraient souhaité que les programmes puissent concerner plusieurs ministères mais cette hypothèse n'a pas été retenue eu égard aux risques de confusion, voire même de paralysie de l'action, qui étaient susceptibles d'en découler. C'est 
pourquoi, au nom d'une logique de responsabilisation, la règle posée par la loi est que les crédits ouverts sont mis à la disposition des ministres et donc placés sous la responsabilité de chacun d'entre eux; ainsi, à chaque programme correspond un seul responsable. Par ailleurs, un responsable de programme est désigné au sein du ministère. Ces programmes sont ensuite découpés en Budgets opérationnels de programme $(B O P)$ avec à leur tête un responsable. Ces BOP sont eux-mêmes susceptibles d'être divisés en Unités opérationnelles de programme (UOP) pilotés là encore par un responsable chargé de réaliser les objectifs conjointement fixés avec le responsable du BOP qui a préalablement déterminé ses propres objectifs en concertation avec le responsable du programme. On appelle cette discussion entre responsables des différents niveaux un « dialogue de gestion ».

\section{La loi de règlement : un outil de contrôle de la gestion}

La logique de contrôle de gestion dont est porteuse la LOLF et dans laquelle se trouve placée la décision financière publique implique nécessairement un retour sur les résultats, et par conséquent un contrôle et une évaluation de l'efficacité des actions. C'est à ce titre que la loi de règlement (LR) fait l'objet d'une revalorisation importante et qu'à travers elle, se trouve organiquement consacré ce que l'on peut à notre sens qualifier de "contrôle parlementaire professionnalisé des finances de l'Etat ». En effet, outre que ses fonctions sont précisées et élargies, la LR est enrichie d' informations telles que les parlementaires devraient y trouver un véritable outil de contrôle pour une analyse et une évaluation des résultats, un outil les conduisant à intégrer un cadre de pensée très proche de celui du chef d'entreprise .

\section{Des fonctions précisées qui empruntent à la logique financière des entreprises}

Si la loi de règlement arrête le montant définitif des recettes et des dépenses du budget ainsi que le résultat budgétaire de l'année, elle fixe également le montant définitif des ressources et des charges de trésorerie ayant concouru à la réalisation de l'équilibre financier de l'année correspondante, présenté dans un tableau de financement .

D'autre part, la loi de règlement intervient dans le processus d'établissement de la comptabilité générale de l'Etat. Elle approuve en effet le compte de résultat de l'exercice, établi à partir des ressources et des charges constatées, l'affecte au bilan qu'elle doit approuver ainsi que ses annexes, étant sur tous ces points très proche d'une véritable logique d'entreprise. 


\section{La loi de règlement : un outil de contrôle professionnalisé de la gestion financière}

La décision budgétaire étant orientée vers les résultats, il va de soi que le Parlement doit disposer d'un document suffisamment complet pour lui permettre de juger de la qualité de la réalisation des prévisions; il s'agit là d'une exigence parfaitement banale du point de vue du contrôle de la gestion. Cette logique se retrouve dans la masse très importante des informations financières fournies aux parlementaires en annexe du projet de loi de règlement et par lesquelles ceux-ci se trouvent placés en quelque sorte dans la situation qui est celle du manager d'une entreprise ou de l'expert chargé d'effectuer une analyse financière.

\section{Autonomie et responsabilisation des administrations}

\section{La responsabilisation des gestionnaires par la fongibilité des crédits}

Le dispositif institué par la loi , qui consiste dans la globalisation des crédits et leur fongibilité à l'intérieur des programmes, devrait être un facteur de transformation essentiel de la gestion publique et du fonctionnement général de l'administration y compris des objectifs et des raisons d'être de ses corps de contrôle.

En effet, avec la globalisation et la fongibilité des crédits, le gestionnaire public est doté d'une plus grande autonomie. Il réalise comme il l'entend le programme dont il a la charge et, indiscutablement, ses marges de manœuvre sont très larges ; il peut redéployer les crédits à son gré entre les titres; il décide quelle doit être la meilleure répartition des dépenses ; il a également la faculté de transformer des crédits de fonctionnement en crédits d'investissement et inversement ( on retrouve ainsi l'extension des autorisations d'engagement /crédits de paiement aux dépenses de fonctionnement). On rappellera toutefois que la fongibilité des crédits ne s'étend pas aux dépenses de personnel. Des crédits de personnel peuvent être utilisé pour financer d'autres dépenses mais l'inverse n'est pas possible, c'est pourquoi l'on parle de fongibilité asymétrique.

Il y a bien entendu une contrepartie. Elle consiste dans la responsabilité qui est celle des gestionnaires vis à vis des objectifs poursuivis et dans leur engagement à devoir réaliser les résultats fixés ; ces derniers doivent ainsi rendre compte de leur gestion et produire un rapport annuel de performances. Des indicateurs permettent d'évaluer la qualité de la gestion accomplie.

\section{Le contrôle de gestion et la mesure de la performance}

La comptabilité publique, ici celle qui concerne l'Etat, se trouve profondément modifiée dans sa philosophie. Les dispositifs prévus comme la logique générale qui sous-tend la loi annoncent en effet une évolution orientée vers une plus grande 
maîtrise de la gestion publique, avec pour ce faire une modélisation de celle-ci sur la gestion privée. A cet égard, la création de systèmes d'information budgétaire et comptable au sein des administrations en vue de permettre de mesurer la performance et de contrôler la gestion des services participe pleinement de cet objectif. Sans compter que privilégiant l'évaluation de l'efficacité et de la performance de la dépense publique, une telle conception amène à reconsidérer les objectifs assignés à la fonction de contrôle.

On précisera encore que le texte opère une distinction nette entre deux notions , celle de comptabilité budgétaire et celle de comptabilité générale de l'Etat et que c'est de la deuxième qu'il entend faire un instrument de contrôle de la gestion et de la performance. Ainsi, si les opérations budgétaires sont établies en comptabilité de caisse tant en ce qui concerne les prévisions que l'exécution, la comptabilité générale de l'Etat est fondée quant à elle sur le principe de la constatation des droits et obligations. Les opérations sont donc prises en compte au titre de l'exercice auquel elles se rattachent, indépendamment de leur date de paiement ou d'encaissement et, en cela, les règles applicables à la comptabilité générale de l'Etat ne se distinguent de celles applicables aux entreprises (plan comptable général des entreprises) qu'au regard des spécificités de son action. La LOLF institue de la sorte un passage d'une comptabilité de flux à une comptabilité patrimoniale, d'une comptabilité de caisse à une comptabilité d'exercice.

Tout aussi proche de la comptabilité d'entreprise, le compte général de l'Etat qui est joint au projet de loi de règlement, comprend la balance générale des comptes, le compte de résultat, le bilan et ses annexes, et une évaluation des engagements hors bilan de l'Etat. Il est accompagné d'un rapport de présentation, qui indique notamment les changements des méthodes et des règles comptables appliqués au cours de l'exercice .

A première vue, la solution choisie, celle d'une double modalité des enregistrements, n'est pas la plus simple; en fait ,le législateur a entendu traiter différemment les opérations budgétaires et les opérations comptables car il a jugé, d'une part qu'un enregistrement aux encaissements correspond mieux à la réalité de l'exécution du budget et assure une meilleure lisibilité immédiate, d'autre part qu'une comptabilité d'exercice est plus appropriée à la mise en place d'un contrôle de gestion, à une évaluation des performances.

On ajoutera encore que selon la loi, les comptes de l'Etat doivent être réguliers, sincères et donner une image fidèle de son patrimoine et de sa situation financière. C'est la Cour des comptes qui a la charge de certifier les comptes de l'Etat ${ }^{5}$. 


\section{Streszczenie}

Reforma finansów publicznych we Francji, wprowadzona na mocy ustawy z 1 sierpnia 2001 r. (LOLF), która stała się swego rodzaju „konstytucją finansową”, dokonała głębokich przemian we francuskim prawie budżetowym. LOLF wpisuje się w historyczny proces reformy finansów publicznych, w proces polityczno-socjologiczny, a także proces międzynarodowych przemian publicznych systemów finansowych. Zawiera strategiczną koncepcję działań wykonywanych przez państwo i podejmowania decyzji dotyczących finansów publicznych. Dzięki niej możliwe jest unowocześnienie struktur politycznych i administracyjnych, a w konsekwencji przystosowanie państwa do nowych uwarunkowań gospodarczych i socjologicznych. 